OITS 762.1

May 2005

\title{
Parton and Hadron Correlations in Jets
}

\author{
Rudolph C. Hwa ${ }^{1}$ and Zhiguang Tan ${ }^{1,2}$ \\ ${ }^{1}$ Institute of Theoretical Science and Department of Physics \\ University of Oregon, Eugene, OR 97403-5203, USA \\ ${ }^{2}$ Institute of Particle Physics, Hua-Zhong Normal University, Wuhan 430079, P. R. China
}

\begin{abstract}
Correlation between shower partons is first studied in high $p_{T}$ jets. Then in the framework of parton recombination the correlation between pions in heavy-ion collisions is investigated. Since thermal partons play very different roles in central and peripheral collisions, it is found that the correlation functions of the produced hadrons behave very differently at different centralities, especially at intermediate $p_{T}$. The correlation function that can best exhibit the distinctive features is suggested. There is not a great deal of overlap between what we can calculate and what has been measured. Nevertheless, some aspects of our results compare favorably with experimental data.

PACS numbers: 25.75.Gz, 25.75.Dw
\end{abstract}




\section{Introduction}

Correlations between hadrons produced in jets in heavy-ion collisions have become a subject of intense study recently [1]-[5]. There are many ways to describe the nature of the correlations, some using triggers, others giving correlations without triggers. So far no clear understanding of the properties of dihadron correlations has emerged. Satisfactory theoretical description of the problem is lagging even farther behind. In this paper we offer a theoretical view of some aspect of the correlations among partons and hadrons in connection with jets. Because we treat only a limited aspect of the dihadron distributions (notably only $\pi^{+} \pi^{+}$correlation), we cannot make exhaustive comparisons with the data that have their own limitations, such as the lack of particle identification in many cases. Nevertheless, our results illuminate some important features of the nature of the correlations.

For experiments at the Relativistic Heavy Ion Collider (RHIC) the relevant range of transverse momenta $\left(p_{T}\right)$ of the detected hadrons in which the correlations are probed is between 0.5 and $9 \mathrm{GeV} / \mathrm{c}$. Since no calculation from first principles is reliable in the intermediate $p_{T}$ range, any theoretical treatment of correlations can only be done in the framework of some phenomenological model. The parton recombination model has been successful in describing the single-particle inclusive distributions in $\mathrm{Au}+\mathrm{Au}[6]$ as well as $\mathrm{d}+\mathrm{Au}$ collisions [7, 8]. Indeed, it has already been applied to the study of some aspects of dihadron correlations [9]. We shall extend that study and examine in more detail various features of the correlations, not only among the produced hadrons, but also among the partons before they hadronize.

There are limitations to what we can do here. We shall continue to use the onedimensional (1D) formulation of parton recombination, which offers a transparent display in 
analytical forms the various components that contribute to dihadron correlations, without any thing hidden in an elaborate code. Focusing only on the $p_{T}$ variable in the $1 \mathrm{D}$ treatment means that we cannot consider the dependencies on the pseudorapidity $(\eta)$ and azimuthal angle $(\phi)$ separations between the two detected hadrons. That shortcoming will be overcome in a separate investigation. But by not being distracted by the behavior in $\Delta \eta$ and $\Delta \phi$ variables, we can examine more closely the separate roles that thermal and shower partons play in the correlation problem, when only the collinear momenta are considered. Experimentally, they correspond to the magnitudes of the associated particle momenta. Even with that simplification we have not considered all charged particles, which are what the experiments measure without particle identification. To gain an overview of the general properties of the correlation functions, the hadron pair $\pi^{+} \pi^{+}$is sufficient and more transparent in providing a rich display of various features.

Our basic assumption in this work is that the shower partons are emitted independently in a jet except for the momentum constraint that the sum of the momentum fractions of all partons cannot exceed 1. This assumption is only a working hypothesis in this study and is a reasonable starting point of our investigation of correlations in order to form a base line, from which the effect of dynamical correlation can be differentiated in the future. Whatever hadronic correlation that we shall calculate in this paper therefore follows from the kinematical correlation of the shower partons in a jet. The dynamical independence of the shower partons may perhaps be approximately correct at small momentum fractions where many shower partons are likely to be present. At larger momentum fractions the kinematical constraint is important and is suitably taken into account. Since the distributions of the shower partons have been determined from the fragmentation functions (FFs) with the 
same assumption of dynamical independence [10], we are in that respect merely being selfconsistent in our study of their correlation here. Since the single-particle distributions that we have calculated using those distributions of the shower partons are in good agreement with the data [6], there is indirect evidence that our assumption may not be too far from reality.

Furthermore, another test of the reliability of the shower parton distributions (SPDs) has been carried out in [11], where the fragmentations of $u$ and $d$ quarks to $p+\bar{p}$ have been calculated using the SPDs for recombination into a proton or antiproton. The resultant FFs agree with the parametrization of KKP [12] over 4 orders of magnitude within a factor of 2 , which is roughly the margin of error made in the KKP parametrization of the data on $p+\bar{p}$ production [11]. Because of that error in [12], the work on the proton FF is not included in [10], but it is nevertheless a demonstration that the assumption of independent shower partons except for kinematical constraint can lead to sensible result in the calculation of the proton FF, which has not been done in any other approach to hadronization, as far as we know. In this paper we continue to use that assumption to calculate the hadronic correlations in heavy-ion collisions.

We shall first examine the single-particle distributions for $\mathrm{Au}+\mathrm{Au}$ collisions at all centralities. The parameters determined can then be used for subsequent studies of correlations among partons and hadrons. In Sec. 3 the correlation among the shower partons is calculated both for jets in vacuum and for jets produced in heavy-ion collisions (HIC). They behave quite differently. Then we study in Sec. 4 the correlation among hadrons, for which the role of thermal partons becomes important in central collisions, but not as much in peripheral collisions. In Sec. 5 our results are compared with existing experimental data in areas where 
such comparisons are possible.

\section{Single-Particle Distributions at all Centralities}

In [6] the single-particle distribution of hadrons produced in $\mathrm{Au}+\mathrm{Au}$ collisions at $200 \mathrm{GeV}$ is studied for $0-10 \%$ centrality only. We now complete the picture by considering all other centralities, thereby determining the corresponding parameters for the thermal partons and the value of $\xi$ that describes the average number of hard partons emerging from the dense medium to hadronize. This also gives us the opportunity to reiterate the basic equations for parton recombination.

The invariant inclusive distribution for a produced meson with momentum $p$ is $[6]$

$$
p \frac{d N_{\pi}}{d p}=\int \frac{d q_{1}}{q_{1}} \frac{d q_{2}}{q_{2}} F_{j j^{\prime}}\left(q_{1}, q_{2}\right) R_{\pi}\left(q_{1}, q_{2}, p\right)
$$

where the recombination function $(\mathrm{RF})$ for a pion is [13]

$$
R_{\pi}\left(q_{1}, q_{2}, p\right)=\frac{q_{1} q_{2}}{p^{2}} \delta\left(\frac{q_{1}}{p}+\frac{q_{2}}{p}-1\right)
$$

The distribution for two partons, $j$ and $j^{\prime}$, that are to recombine has three components

$$
F_{j j^{\prime}}=\mathcal{T} \mathcal{T}+\mathcal{T} \mathcal{S}+\mathcal{S S}
$$

where the thermal parton distribution is

$$
\mathcal{T}(q)=C q e^{-q / T}
$$

and the shower parton in $\mathcal{T S}$ has the distribution

$$
\mathcal{S}(q)=\xi \sum_{i} \int d k k f_{i}(k) S_{i}^{j}(q / k)
$$


whereas the two shower partons in the same jet $(\mathcal{S S})$ have the correlated distribution

$$
\begin{gathered}
(\mathcal{S S})\left(q_{1}, q_{2}\right)=\xi \sum_{i} \int d k k f_{i}(k)\left\{S_{i}^{j}\left(\frac{q_{1}}{k}\right), S_{i}^{j^{\prime}}\left(\frac{q_{2}}{k-q_{1}}\right)\right\} \\
\left\{S_{i}^{j}\left(x_{1}\right), S_{i}^{j^{\prime}}\left(\frac{x_{2}}{1-x_{1}}\right)\right\}=\frac{1}{2}\left[S_{i}^{j}\left(x_{1}\right) S_{i}^{j^{\prime}}\left(\frac{x_{2}}{1-x_{1}}\right)+S_{i}^{j}\left(\frac{x_{1}}{1-x_{2}}\right) S_{i}^{j^{\prime}}\left(x_{2}\right)\right] .
\end{gathered}
$$

The distribution $f_{i}(k)$ of hard parton $i$ at midrapidity in $\mathrm{Au}+\mathrm{Au}$ collisions is given in [14]. The integral over $k$ is from $k_{\text {min }}=3 \mathrm{GeV} / \mathrm{c}$ to an upper limit which we shall set at $40 \mathrm{GeV} / \mathrm{c}$. $S_{i}^{j}$ is a matrix of shower parton distributions (SPD) that have been determined in [10]. The form of the two SPDs, given in Eq. (7), symmetrizes the order of emission and guarantees the momentum conservation condition $x_{1}+x_{2} \leq 1$, apart from which the shower partons are assumed to be independent. Thus the correlation between the partons is essentially kinematical, as it is treated here, although other correlations of more dynamical origin can be considered later when the need arises.

The shower partons can either recombine among themselves, or recombine with the thermal partons. In the former case the process reproduces the FF

$$
x D_{i}^{\pi}(x)=\int \frac{d x_{1}}{x_{1}} \frac{d x_{2}}{x_{2}}\left\{S_{i}^{j}\left(x_{1}\right), S_{i}^{j^{\prime}}\left(\frac{x_{2}}{1-x_{1}}\right)\right\} R_{\pi}\left(x_{1}, x_{2}, x\right) .
$$

Indeed, this is the equation from which the SPDs are determined in the first place [10]. Thus the $\mathcal{S S}$ term in Eq. (3), when substituted into Eq. (1), yields the fragmentation component

$$
p \frac{d N_{\pi}^{\mathcal{S S}}}{d p}=\xi \sum_{i} \int d k k f_{i}(k) \frac{p}{k} D_{i}^{\pi}\left(\frac{p}{k}\right)
$$

Note that because the two shower partons are from the same jet, Eq. (9) depends linearly on $\xi f_{i}(k)$, which is the same dependence that the $\mathcal{T S}$ component has. Hence, when all three terms in $F_{j j^{\prime}}$ and the RF $R_{\pi}$ are substituted into Eq. (1), we have, in the notation $p=p_{T}$,

$$
\frac{d N_{\pi}}{p d p}=\frac{C^{2}}{6} e^{-p / T}+\frac{\xi}{p} \sum_{i j} \int d k k f_{i}(k)\left[\frac{1}{k} D_{i}^{\pi}\left(\frac{p}{k}\right)+\frac{C}{p^{2}} \int d q_{1} S_{i}^{j}\left(\frac{q_{1}}{k}\right)\left(p-q_{1}\right) e^{-\left(p-q_{1}\right) / T}\right] .
$$


This is the basic formula for the inclusive distribution of pion. The hard parton $i$ is summed over all relevant species, while $j$ is summed over the two possible constituent quark species of the pion. The quark $j$ picks up a partner from the thermal environment to form the pion. That $\mathcal{T S}$ component turns out to be dominant in the intermediate $p_{T}$ region $\left(3<p_{T}<9\right.$ $\mathrm{GeV} / \mathrm{c}$ ) in central $\mathrm{Au}+\mathrm{Au}$ collisions, and is responsible for the high $p / \pi$ ratio, when the production of proton is considered in a similar way [6].

We now apply Eq. (10) to non-central Au+Au collisions. The hard parton distribution $f_{i}(k)$ is scaled down from central collisions by the average number of binary collisions $\left\langle N_{\text {coll }}\right\rangle$. Our procedure is to fit the low- $p_{T}$ data (for $p_{T}<2 \mathrm{GeV} / \mathrm{c}$ ) by the first term that arises from $\mathcal{T} \mathcal{T}$ recombination, thereby determining $C$ and $T$ for each centrality. The only remaining parameter, $\xi$, is then determined by the overall normalization of the spectrum at each centrality so that the shape of the large $p_{T}$ dependence is a prediction of Eq. (10). The data used are the $\pi^{0}$ spectra from PHENIX that extend up to $p_{T}=10 \mathrm{GeV} / \mathrm{c}$ [15]. We, however, calculate the $\pi^{+}$distribution with $j=u$ and $\bar{d}$, since our formalism does not distinguish the two. Figure 1 shows the result of our calculation for four representative centralities, although we have treated all nine centralities, for which the parameters are shown in Fig. 2. Evidently, the spectra are well fitted at all centralities. The inverse slope $T$ shows no significant dependence on centrality; the common value may be set at

$$
T=0.305 \mathrm{GeV} / \mathrm{c} .
$$

The value of $\xi$ increases from 0.09 at $0-10 \%$ to 0.97 at $80-92 \%$ centrality. Thus $\mathrm{Au}+\mathrm{Au}$ collision at $80-92 \%$ is essentially equivalent to $p p$ collision, since the suppression factor due to energy loss is nearly 1, i.e., no jet quenching. The value of $C$ decreases from $21.8(\mathrm{GeV} / \mathrm{c})^{-1}$ 
at $0-10 \%$ to $2.3(\mathrm{GeV} / \mathrm{c})^{-1}$ at $80-92 \%$. The thermal component in peripheral collision is therefore much reduced, as expected.

The contributions from the various components are shown in Fig. 3 for $0-10 \%$ and 80-92\% centralities. It is clear that at $0-10 \%$ the component from thermal-shower recombination is dominant in the $3<p_{T}<9 \mathrm{GeV} / \mathrm{c}$ range, but at $80-92 \%$ that component is lower than both thermal-thermal and shower-shower components. Indeed, $\mathcal{S S}$ dominates for all $p_{T}>3$ $\mathrm{GeV} / \mathrm{c}$; it means that the fragmentation model is quite adequate for $p p$ collisions in that $p_{T}$ range.

\section{Correlation of Partons in Jets}

Before we consider the correlation between hadrons produced in jets, let us first study the simpler problem of correlation between partons in jets. Although the latter is not measurable, it provides a striking view of the difference between jets produced in vacuum, as in $e^{+} e^{-}$ annihilation, and jets produced in HIC where the hard parton momentum is not fixed.

To define our notation, let $\rho_{1}(1)$ and $\rho_{2}(1,2)$ be the single-particle and two-particle distributions, and we define the two-particle correlation function as

$$
C_{2}(1,2)=\rho_{2}(1,2)-\rho_{1}(1) \rho_{1}(2)
$$

Later, when we apply this formula to hadron correlation, the experimentally measurable quantities are

$$
\rho_{1}(1)=\frac{d N_{\pi_{1}}}{p_{1} d p_{1}}, \quad \rho_{2}(1,2)=\frac{d N_{\pi_{1} \pi_{2}}}{p_{1} d p_{1} p_{2} d p_{2}}
$$


The normalized correlation function is

$$
K_{2}(1,2)=\frac{C_{2}(1,2)}{\rho_{1}(1) \rho_{1}(2)}=r_{2}(1,2)-1
$$

where

$$
r_{2}(1,2)=\frac{\rho_{2}(1,2)}{\rho_{1}(1) \rho_{1}(2)}
$$

For reasons that will become evident below, it is useful to consider the ratio

$$
G_{2}(1,2)=\frac{C_{2}(1,2)}{\left[\rho_{1}(1) \rho_{1}(2)\right]^{1 / 2}},
$$

a quantity that is similar to the Pearson's correlation [16] under certain assumptions, and is closely related to a quantity considered in [2]

\subsection{Two Shower Partons in a Jet in Vacuum}

When a hard parton $i$ with any fixed large momentum $k$ fragments in vacuum to a shower of semi-hard and soft partons, we denote the single- and two-parton distributions in terms of the momentum fractions $x_{1}$ and $x_{2}$ by

$$
\begin{gathered}
\rho_{1}(1)=S_{i}^{j}\left(x_{1}\right), \\
\rho_{2}(1,2)=(S S)_{i}^{j j^{\prime}}\left(x_{1}, x_{2}\right),
\end{gathered}
$$

where the RHS of Eq. (18) is to be identified with Eq. (7). For definiteness we consider $j=u$ and $j^{\prime}=\bar{d}$. For $i=g$ we get for $r_{2}(1,2)$ a 2D distribution as shown in Fig. 4 . Other distributions with $i=u$ or $\bar{d}$ are very similar.

Since $r_{2}(1,2)=1$ implies no correlation, we see from Fig. 4 that the two shower partons are uncorrelated when $x_{1} \approx x_{2} \approx 0$, but are highly correlated at higher $x_{i}$. Kinematical 
constraint due to momentum conservation forces $r_{2}(1,2)$ to vanish at $x_{1}+x_{2}=1$. It approaches zero faster, as $x_{1}=x_{2} \rightarrow 0.5$, than it does when $x_{2}$ is fixed and small, as $x_{1} \rightarrow 1-x_{2}$. The implication is that if these two partons are to recombine to form a pion at $x=x_{1}+x_{2}$, there is more contribution from the asymmetric pairing of $x_{1}$ and $x_{2}$ than from the unnecessarily stringent requirement that $x_{1}=x_{2}=x / 2$.

\subsection{Two Shower Partons in a Jet in HIC}

For each event in HIC with a high $p_{T}$ jet, we can calculate the two-parton distribution as above. However, the hard parton momentum $k$ cannot be fixed in an experiment. The distribution in the shower parton momenta $q_{1}$ and $q_{2}$, when averaged over all events, necessitates an integration over $k$ and a sum over all $i$. The single-parton distribution

$$
\rho_{1}(1)=\mathcal{S}^{j}\left(q_{1}\right)
$$

is given by Eq. (5), while the two-parton distribution

$$
\rho_{2}(1,2)=(\mathcal{S S})^{j j^{\prime}}\left(q_{1}, q_{2}\right)
$$

is given by Eqs. (6) and (7). Some details in the calculation need to be explained. When $i$ in Eq. (6) is $g$, then $S_{i}^{j} S_{i}^{j^{\prime}}=G G$, but when $i=u$, say, then $S_{i}^{j} S_{i}^{j^{\prime}}=K L$, where $G, K$ and $L$ are SPDs initiated by gluon, valence quark and sea quark, respectively, in the notation of [10]. It turns out that the gross features of the results do not depend sensitively on these details.

For $\mathrm{Au}+\mathrm{Au}$ collisions at $200 \mathrm{GeV}$ we have calculated $r_{2}(1,2)$. The results for most central and most peripheral collisions are shown in Fig. 5. The main feature is that $r_{2}(1,2)$ is large 
and increases with $q_{1}$ and $q_{2}$. That is markedly different from Fig. 4. The reason is that $\rho_{1}(1) \rho_{1}(2)$ depends quadratically on $\xi f_{i}(k)$, whereas $\rho_{2}(1,2)$ depends on it linearly. The probability $\xi f_{i}(k)$ for a hard parton to be produced at $k$ in HIC is small in central collisions, and is even smaller in peripheral collisions (although $\xi$ is larger) because $f_{i}(k)$ scales with $\left\langle N_{\text {coll }}\right\rangle$. Thus the probability of producing two partons in a jet is much higher than the product of the probabilities of producing the two partons separately. The latter does not require the two partons to be produced in two jets in the same event. For experiments at RHIC we may assume that events with two jets in the same hemisphere never occur, since they are so rare. Another way of interpreting $r_{2}(1,2)$ is to write it as

$$
r_{2}(1,2)=\rho_{c}(2 \mid 1) / \rho_{1}(2)
$$

where $\rho_{c}(2 \mid 1)$ is the conditional probability of finding a parton at $q_{2}$ given that a parton is at $q_{1}$

$$
\rho_{c}(2 \mid 1)=\frac{\rho_{2}(1,2)}{\rho_{1}(1)}
$$

Thus $\rho_{c}(2 \mid 1)$ is like the distribution of a particle associated with a trigger at $q_{1}$. The largeness of the ratio in Eq. (21) means that it is far more likely for a shower parton to be found at $q_{2}$ in association with another parton already found at $q_{1}$ than to find a parton at $q_{2}$ independently.

The two surfaces in Fig. 5 have essentially the same shape. The peripheral case is higher because the corresponding $\xi f_{i}(k)$ is smaller. In Fig. 4 the hard parton momentum $k$ is held fixed and the probability of having such a parton is 1 , while in Fig. $5 k$ is integrated up to $40 \mathrm{GeV} / \mathrm{c}$ so that at higher $q_{1}$ and $q_{2}$ there is no kinematical cut-off, albeit the probability $f_{i}(k)$ of producing a hard parton at higher $k$ is greatly suppressed, which only makes $r_{2}(1,2)$ higher. 
The study in this section may seem academic, since shower partons are not directly observable. However, those shower partons are the main constituents that contribute to the formation of pions that we shall treat in the following section. Their properties are therefore important to help us understand the features that we shall find about dihardron correlations. It should be emphasized that no free parameters have been used in this section, or in the section to follow, apart from $C, T, \xi$ that have been determined in Sec. 2 for the single-particle distributions.

\section{Correlation of Pions in Jets}

For the production of two pions it is necessary to consider the recombination of four partons. The corresponding equation for the two-pion distribution is [9]

$$
\frac{d N_{\pi_{1} \pi_{2}}}{p_{1} p_{2} d p_{1} d p_{2}}=\frac{1}{p_{1}^{2} p_{2}^{2}} \int\left(\prod_{i=1}^{4} \frac{d q_{i}}{q_{i}}\right) F_{4}\left(q_{1}, q_{2}, q_{3}, q_{4}\right) R_{\pi_{1}}\left(q_{1}, q_{3}, p_{1}\right) R_{\pi_{2}}\left(q_{2}, q_{4}, p_{2}\right) .
$$

The partons with momenta $q_{1}$ and $q_{3}$ that form $\pi_{1}$ can be either thermal or shower, as in Eq. (3). Similarly, the same is true with $q_{2}$ and $q_{4}$. Thus $F_{4}$ has the form

$$
F_{4}=(\mathcal{T} \mathcal{T}+\mathcal{S T}+\mathcal{S S})_{13}(\mathcal{T} \mathcal{T}+\mathcal{S T}+\mathcal{S S})_{24}
$$

Among the various possible combinations of terms, it is clear that $(\mathcal{T} \mathcal{T})_{13}(\mathcal{T} \mathcal{T})_{24}$ has nothing to do with hard scattering and contributes to the background. For that reason the terms involving $(\mathcal{T} \mathcal{T})$ are not included in [9], where the background-subtracted distributions of the associated particles are considered. Here we study $C_{2}(1,2)$, which has its own definition of subtraction, so we start out being general. Despite the deceptive appearance of Eq. (24), $F_{4}$ is not factorizable. If it were, its substitution into Eq. (23) would render the factorizable 
form $\rho_{2}(1,2)=\rho_{1}(1) \rho_{2}(2)$, implying no correlation. The nonfactorizability of $F_{4}$ is rooted in the correlations among the various shower partons. The correlation between two shower partons in $(\mathcal{S S})_{13}$ or $(\mathcal{S S})_{24}$ has already been considered in connection with Eq. (20), which is shown explicitly in Eqs. (6) and (7). However, those are not the correlations that render $F_{4}$ nonfactorizable. It is the correlation between the partons in $(1,3)$ with the partons in $(2,4)$ that gives rise to nonvanishing $C_{2}(1,2)$. More specifically, because all shower partons in Eq. (24) originate from the same hard parton at $k$, there is only one $\xi f_{i}(k)$ factor for all $\mathcal{S S} \cdots$ terms. Thus, for example, in $(\mathcal{S T})_{13}(\mathcal{S T})_{24}$ the $(\mathcal{S S})\left(q_{1}, q_{2}\right)$ term is exactly as expressed in Eq. (6). In $(\mathcal{S T})_{13}(\mathcal{S S})_{24}$ we can append $R_{\pi_{2}}\left(q_{2}, q_{4}, p_{2}\right)$ and make use of Eq. (8) to convert $(\mathcal{S S})_{24} R_{\pi_{2}}$ to the FF $D^{\pi_{2}}$, so that the $(\mathcal{S T})(\mathcal{S S})$ contributions to $\rho_{2}(1,2)$ is explicitly

$$
\begin{aligned}
\frac{d N_{\pi_{1} \pi_{2}}^{s t s s}}{p_{1} p_{2} d p_{1} d p_{2}}= & \frac{\xi}{p_{1}^{3} p_{2}} \sum_{i, i^{\prime}, i^{\prime \prime}} \int d k k f_{i}(k) \int d q_{1} \mathcal{T}\left(p_{1}-q_{1}\right) \\
& \cdot \sum_{j} \frac{1}{2}\left[S_{i^{\prime}}^{j}\left(\frac{q_{1}}{k}\right) \frac{1}{k-q_{1}} D_{i^{\prime \prime}}^{\pi_{2}}\left(\frac{p_{2}}{k-q_{1}}\right)+S_{i^{\prime}}^{j}\left(\frac{q_{1}}{k-p_{2}}\right) \frac{1}{k} D_{i^{\prime \prime}}^{\pi_{2}}\left(\frac{p_{2}}{k}\right)\right]
\end{aligned}
$$

which is not factorizable. For definiteness we shall hereafter consider $\pi_{1}$ and $\pi_{2}$ to be both $\pi^{+}$. Then the sum in $j$ is over $u$ and $\bar{d}$. If $i=g$, then $i^{\prime}, i^{\prime \prime}=g$; if $i=V(u$ or $\bar{d})$, then $\left(i^{\prime}, i^{\prime \prime}\right)=(V, S)$ or $(S, V)$ where $S$ denotes sea; if $i=S$, then $i^{\prime}, i^{\prime \prime}=S$ also.

For completeness, let us write out the two other combinations of terms. For $(\mathcal{S T})_{13}(\mathcal{S T})_{24}$ we have

$$
\begin{aligned}
\frac{d N_{\pi_{1} \pi_{2}}^{s t s t}}{p_{1} p_{2} d p_{1} d p_{2}}= & \frac{\xi}{p_{1}^{3} p_{2}^{3}} \sum_{i, i^{\prime}, i^{\prime \prime}} \int d k k f_{i}(k) \int d q_{1} d q_{2} \mathcal{T}\left(p_{1}-q_{1}\right) \mathcal{T}\left(p_{2}-q_{2}\right) \\
& \sum_{j j^{\prime}}\left\{S_{i^{\prime}}^{j}\left(\frac{q_{1}}{k}\right), S_{i^{\prime \prime}}^{j^{\prime}}\left(\frac{q_{2}}{k-q_{1}}\right)\right\},
\end{aligned}
$$


where $j$ and $j^{\prime}$ can be either $u$ or $\bar{d}$. For $(\mathcal{S S})_{13}(\mathcal{S S})_{24}$ we have

$$
\frac{d N_{\pi_{1} \pi_{2}}^{s s s s}}{p_{1} p_{2} d p_{1} d p_{2}}=\frac{\xi}{p_{1} p_{2}} \sum_{i, i^{\prime}, i^{\prime \prime}} \int d k k f_{i}(k)\left\{\frac{1}{k} D_{i^{\prime}}^{\pi_{1}}\left(\frac{p_{1}}{k}\right), \frac{1}{k-p_{1}} D_{i^{\prime \prime}}^{\pi_{2}}\left(\frac{p_{2}}{k-p_{1}}\right)\right\} .
$$

In all three equations above $i, i^{\prime}$ and $i^{\prime \prime}$ follow the same rule stated at the end of the previous paragraph. For simplicity let us use the symbolic form for these three equations as follows: $[(\mathcal{S T}) \mathcal{D}],[(\mathcal{S T})(\mathcal{S T})]$ and $[\mathcal{D D}]$, respectively. The parentheses imply the contents are to recombine; the square brackets signify that the shower partons inside all arise from one hard-parton jet.

It is appropriate at this point to compare our description of two-particle distributions with the correlation study of Fries et al (FBM) [17]. Since both approaches are based on the recombination model $[6,18,19]$, there are many similarities, particularly in the separation into various components. Apart from the difference in focus where we consider correlation in $p_{T}$, while FBM studies correlation in $\phi$, the main difference in physics is that we break the hard parton into shower partons, whereas FBM does not. If our $\mathcal{T} \mathcal{S}$ recombination is related qualitatively to their soft-hard $(\mathrm{SH})$ recombination, then the classification of components is essentially the same in the two approaches. Quantitatively, $\mathcal{T S}$ can be significantly different from SH. Moreover, the correlation at the parton level is for us among the shower partons, while for FBM among the soft partons.

In $\rho_{2}(1,2)$ the $(\mathcal{T} \mathcal{T})(\mathcal{T} \mathcal{T})$ term is factorizable, and is cancelled by the thermal terms in $\rho_{1}(1) \rho_{1}(2)$, where $\rho_{1}(1)$ is given by Eq. (10). That corresponds to background subtraction. Other double $\mathcal{T} \mathcal{T}$ terms like $(\mathcal{T} \mathcal{T})(\mathcal{S} \mathcal{T})$ also get cancelled. The surviving terms in $C_{2}(1,2)$ all involve shower partons in each pion. We group them in four clusters of terms

$$
C_{2}(1,2)=C_{2}^{s t s t}(1,2)+C_{2}^{s t s s}(1,2)+C_{2}^{s s s t}(1,2)+C_{2}^{s s s}(1,2)
$$


where

$$
\begin{gathered}
C_{2}^{\text {stst }}(1,2)=[(\mathcal{S T})(\mathcal{S T})]_{12}-(\mathcal{S T})_{1} *(\mathcal{S T})_{2} \\
C_{2}^{s t s s}(1,2)=[(\mathcal{S T})(\mathcal{D})]_{12}-(\mathcal{S T})_{1} *(\mathcal{D})_{2} \\
C_{2}^{s s s t}(1,2)=[\mathcal{D}(\mathcal{S T})]_{12}-(\mathcal{D})_{1} *(\mathcal{S T})_{2} \\
C_{2}^{s s s s}(1,2)=[\mathcal{D} \mathcal{D}]_{12}-(\mathcal{D})_{1} *(\mathcal{D})_{2}
\end{gathered}
$$

The products involving $*$ signify terms from $\rho_{1}(1) \rho_{1}(2)$, so their shower partons have their own hard partons. More precisely, $(\mathcal{S T})_{1} *(\mathcal{S T})_{2}$, for example, has the explicit expression

$$
\begin{gathered}
(\mathcal{S T})_{1} *(\mathcal{S T})_{2}=\left(\frac{\xi}{p_{1}^{3}} \sum_{i j} \int d k k f_{i}(k) \int d q_{1} S_{i}^{j}\left(\frac{q_{1}}{k}\right) \mathcal{T}\left(p_{1}-q_{1}\right)\right) \\
\cdot\left(\frac{\xi}{p_{2}^{3}} \sum_{i j} \int d k^{\prime} k^{\prime} f_{i}\left(k^{\prime}\right) \int d q_{2} S_{i}^{j}\left(\frac{q_{2}}{k^{\prime}}\right) \mathcal{T}\left(p_{2}-q_{2}\right)\right)
\end{gathered}
$$

Clearly, this is very different from $[(\mathcal{S T})(\mathcal{S T})]_{12}$, written out in full in Eq. (26). Thus $C_{2}^{s t s t}(1,2)$ does not vanish, in general, and likewise for the other terms in Eq. (28).

Regarding Eqs. (27) and (32) that involve FFs only, it is relevant to compare them to the medium modified dihadron FF studied in [20]. In our formulation of hadron production in HIC the medium effect is taken into account by considering $(\mathcal{T} \mathcal{T})$ and $(\mathcal{T S})$ recombination, leaving the $(\mathcal{S S})$ recombination to be unaffected by the medium except for the hard parton distribution $f_{i}(k)$. Thus in Eqs. (9) and (27) the FF in vacuum is used. The contribution of $C_{2}^{s s s s}(1,2)$ to the observed correlation cannot be measured separately, except in $p p$ collisions or in very peripheral $A A$ collisions where the medium effect is negligible. In the fragmentation model modification of the FF is the only way to take into account of the medium effect, 
whereas in the recombination model fragmentation in vacuum is unimportant compared to thermal-shower recombination. For these reasons it is inappropriate to compared Eq. (27) for central nuclear collisions with the medium modified dihadron fragmentation process considered in [20] without including Eqs. (25) and (26) also.

We have calculated all the quantities in Eqs. (28) - (32). The results cannot easily be displayed because each term decreases rapidly with $p_{1}$ and $p_{2}$ and the various clusters of terms, $C_{2}^{\alpha},\{\alpha=s t s t, \ldots\}$, can be positive and negative, so that neither linear nor log plots are appropriate. To give a sense of the complication, we show in Fig. 6(a) the result for $\pi^{+} \pi^{+}$ correlation in central $\mathrm{Au}+\mathrm{Au}$ collisions for the restricted case of $p_{1}=p_{2}=p$ in the range 1.5-10 GeV/c. At $p \approx 2 \mathrm{GeV} / \mathrm{c}$, all $C_{2}^{\alpha}$ become negative, resulting in a $\operatorname{dip}$ in $C_{2}$. There is no such fine structure for peripheral collisions (80-92\%), so a semi-log plot can exhibit the behaviors of $C_{2}^{\alpha}$ in the entire range up to $p=10 \mathrm{GeV} / \mathrm{c}$, as shown in Fig. 6(b). Note that for 0-10\% centrality the terms involving st component is dominant, while for $80-92 \%$ centrality the $\alpha=$ ssss component is dominant. These features are in accord with the properties of single-particle distributions shown in Fig. 3, where thermal-shower and shower-shower components, respectively, are dominant in the two cases.

When $p_{1}$ and $p_{2}$ are different, the two surfaces of $C_{2}(1,2)$ for the two centralities are shown in Fig. 7 in the $2 \mathrm{D}$ ranges where they are positive. The region where $C_{2}(1,2)$ is negative cannot be shown in the semi-log plot. For what can be shown they behave rather similarly. They both are suppressed by about 10 orders of magnitude going from 1 to 10 $\mathrm{GeV} / \mathrm{c}$. A large part of the rapid decrease is due to the power-law suppression that is present even in the single-particle distributions over the same $p_{T}$ range, as can be seen in Fig. 1, though not more than 8 orders of magnitude. Thus the correlation function $C_{2}(1,2)$ is not 
the best quantity to exhibit the properties of correlation.

To de-emphasize the power-law suppression of the hard parton distribution at high $k$ that is contained in $\rho_{1}(1)$ and $\rho_{1}(2)$, we consider the normalized correlation $K_{2}(1,2)$ defined in Eq. (14). When we set $p_{1}=p_{2}=p$, the dependence of $K_{2}(p)$ on $p$ is shown in Fig. 8 for (a) $0-10 \%$ and (b) $80-92 \%$ centrality. In (a) a dip occurs at low $p$, the minimum being at $p=3 \mathrm{GeV} / \mathrm{c}$ with $K_{2}<0$; in (b) there is no dip. The rapid rise at larger $p$ is introduced into $K_{2}(1,2)$ by the division of $C_{2}(1,2)$ by $\rho_{1}(1) \rho_{1}(2)$, which is strongly damped at high $p_{1}$ and $p_{2}$. It is the result of an over-correction. The fact that $K_{2}(80-92 \%) \gg K_{2}(0-10 \%)$ at all $p$ in Fig. 8 is due to the property of $\xi f_{i}(k)$ being much smaller in peripheral collisions compared to central collisions. Since the numerator of $K_{2}(1,2)$ depends linearly on $\xi f_{i}(k)$, but the denominator depends quadratically on it, the peripheral $K_{2}(1,2)$ is therefore much larger.

What we see in Fig. 8 (a) and (b) about the general features of $K_{2}(1,2)$ for the two centralities is very similar to what is shown in Fig. 5 for $r_{2}(1,2)$ for shower partons, recalling that $K_{2}(1,2)=r_{2}(1,2)-1$, as stated in Eq. (14). Although $r_{2}(1,2)$ cannot become negative, it becomes large in Fig. 5 at high $q_{1}$ and $q_{2}$ for both centralities. Of course, hadron correlation is far more complicated than parton correlation. But the common feature that they both become large at large parton and hadron momenta is due to the commonality in the definition of $r_{2}(1,2)$ and $K_{2}(1,2)$, which have $\rho_{1}(1) \rho_{1}(2)$ in the denominators.

It is now clear that we need something that is between $C_{2}(1,2)$ and $K_{2}(1,2)$ so as to have linear dependence on $\xi f_{i}(k)$ in both the numerator and the denominator. That quantity is the ratio $G_{2}(1,2)$, defined in Eq. (16). Figure 8 (c) and (d) shows the dependence of $G_{2}(1,2)$ on $p_{1}=p_{2}=p$ for (c) $0-10 \%$ and (d) $80-92 \%$ centrality. Now, $G_{2}(p)$ is small at large $p$ in 
both cases, and the properties of both can be revealed in linear plots. For $0-10 \%$ centrality there is still a dip at $p=2 \mathrm{GeV} / \mathrm{c}$ with $G_{2}(p)<0$ in its vicinity, as it should, but for $80-92 \%$ centrality $G_{2}(p)$ decreases monotonically with increasing $p$. The normalizations of $G_{2}(p)$ for the two cases are now nearly the same. That can be seen more easily in semi-log plots. In Fig. 9 we show all three correlation function, $C_{2}, K_{2}$ and $G_{2}$, for the two centralities. The gap in Fig. 9(a) is due to the functions being negative in that range. Evidently, we have $C_{2}(0-10 \%) \gg C_{2}(80-92 \%), K_{2}(0-10 \%) \ll K_{2}(80-92 \%)$, but $G_{2}(0-10 \%) \approx G_{2}(80-92 \%)$ in regions where they can be compared. Thus the function $G_{2}(1,2)$ is best for the study of the nature of correlation.

In Fig. 10(a) we show $G_{2}(1,2)$ in $2 \mathrm{D}$ distributions for both centralities in the same linear space. We now can see clearly their differences, knowing that asymptotically they behave similarly without structure. In the ranges of $p_{1}$ and $p_{2}$ plotted $G_{2}(1,2)$ shows negative correlation for $0-10 \%$, but positive correlations for $80-92 \%$ centrality. That difference can be succinctly exhibited in the ratio

$$
R_{C P}^{G_{2}}(1,2)=\frac{G_{2}^{(0-10 \%)}(1,2)}{G_{2}^{(80-92 \%)}(1,2)}
$$

which is shown in Fig. 10(b). Since peripheral Au+Au collisions at 80-92\% centrality is very close to $p p$ collisions, we may regard Fig. 10(b) as the clearest revelation of the difference in dihadron correlation between central $\mathrm{Au}+\mathrm{Au}$ and $p p$ collisions.

The origin of the dip in Fig. 10(b) can be found in Fig. 6 where $C_{2}$ is negative at $p_{1}=p_{2}=p=2 \mathrm{GeV} / \mathrm{c}$ for $0-10 \%$ centrality, but not for $80-92 \%$ centrality. Let us now describe the reasons why $C_{2}^{\alpha}$ are negative in Fig. 6(a), but not in Fig. 6(b). Many factors are involved with competing strengths that can be compared only by concrete calculation, 
but qualitative discussion can provide some insight. Consider Eq. (29) whose two terms are shown explicitly in Eqs. (26) and (33). The former has one factor of $\xi f_{i}(k)$ and two SPDs sharing the same $k$, while the latter has two independent factors of $\xi f_{i}(k)$, each having only one SPD. In the peripheral case $\xi f_{i}(k)$ is very small because $f_{i}(k)$ is scaled down by $\left\langle N_{\text {coll }}\right\rangle$, so $(\mathcal{S T})_{1} *(\mathcal{S T})_{2}$ is always small compared to $[(\mathcal{S T})(\mathcal{S T})]_{12}$ at all $q_{1}$ and $q_{2}$. Hence, $C_{2}^{\text {stst }}(1,2)$ is always positive; the same argument applies to all other $C_{2}^{\alpha}(1,2)$. In the case of central collisions $\xi f_{i}(k)$ becomes large enough to allow other factors to also play some role. When $p_{1}$ and $p_{2}$ are on the lower end of the intermediate range, e.g. $\leq 4 \mathrm{GeV} / \mathrm{c}, q_{1}$ and $q_{2}$ must be even less, but not too small because $\mathcal{T}\left(p_{1}-q_{1}\right)$ and $\mathcal{T}\left(p_{2}-q_{2}\right)$ would be exponentially damped at large $p_{i}-q_{i}$. In other words, if the momentum fraction $x_{i}$ of the shower partons are small, there are not many thermal partons to make up $p_{1}$ and $p_{2}$; on the other hand, if $x_{i}$ are large, the SPDs are suppressed. The hard parton $k$ cannot be large to relieve the latter constraint without suffering the suppression of $f_{i}(k)$. This situation of multiple constraints is easier for $(\mathcal{S T})_{1} *(\mathcal{S T})_{2}$ to meet since there is only one shower parton per jet in Eq. (33), but is much more difficult for $[(\mathcal{S T})(\mathcal{S T})]_{12}$ where the jet momentum must be shared by two shower partons. Thus $[(\mathcal{S T})(\mathcal{S T})]_{12}$ is forced to be smaller than $(\mathcal{S T})_{1} *(\mathcal{S T})_{2}$ in central collisions where $\xi f_{i}(k)$ is not infinitesimal and thermal partons are important in the intermediate $p_{T}$ region.

In the case of $C_{2}^{s s s s}(1,2)$ there is no $\mathcal{T}$, but the demand for additional shower partons plays the same role. It is clear from Eq. (32) that double fragmentation causes $[\mathcal{D D}]_{12}$ to be more suppressed than two single fragmentation $(\mathcal{D})_{1} *(\mathcal{D})_{2}$. Thus $C_{2}^{s s s}(1,2)$ becomes negative so long as $p_{1}$ and $p_{2}$ are not high enough to force the large $k$ to dampen double $\xi f_{i}(k)$ in $(\mathcal{D})_{1} *(\mathcal{D})_{2}$ more severely than the single $\xi f_{i}(k)$ in $[\mathcal{D D}]_{12}$. The other $C_{2}^{\alpha}$ terms behave 
similarly for the same reason, resulting in the sum $C_{2}(1,2)$ to be negative when $p_{1}$ and $p_{2}$ are both less than around $3 \mathrm{GeV} / \mathrm{c}$. The negativity of $C_{2}(1,2)$ should not be surprising, since the ratio $r_{2}(1,2)$ for the shower partons in a jet of fixed $k$ is less than 1 , as shown in Fig. 4. The corresponding $C_{2}(1,2)$ is negative for all $x_{1}$ and $x_{2}$. In the hadronic problem there are in some terms even more shower partons involved, all competing for the limited momentum that the hard parton provides. Fig. 10 exhibits the intricacies of the hadronic correlation even when the basic correlations among the shower partons are kinematical in nature.

\section{Comparison with Experimental Data}

In the foregoing we have studied the correlation of two $\pi^{+}$without using any adjustable parameters. What we have obtained unfortunately cannot be compared directly with current data, because most experiments either do not yet have particle identification, or put emphasis on jets with trigger particles and study the dependencies on $\Delta \phi$ and $\Delta \eta$ of associated particles. There are, however, two areas in which our theoretical calculations come close to the experimental data and are of interest for us to examine them in more detail here.

The first area concerns the experiment in [5], in which data are given for the $p_{T}$ distribution of charged particles associated with trigger in the range $4<p_{T}<6 \mathrm{GeV} / \mathrm{c}$. We can come close to what is measured by calculating

$$
\frac{d N_{\pi^{+}}^{t r-b g}}{p_{2} d p_{2}}=\int_{4}^{6} d p_{1} p_{1} \frac{d N_{\pi^{+} \pi^{+}}^{t r-b g}}{p_{1} p_{2} d p_{1} d p_{2}} / \int_{4}^{6} d p_{1} p_{1} \frac{d N_{\pi^{+}}}{p_{1} d p_{1}}
$$

where $p_{1}$ is regarded as the momentum of the trigger particle, and $p_{2}$ that of the associated particle. The superscript, $t r-b g$, denotes the sample of triggered events with background subtracted. For the integrand in the numerator we use Eq. (23), but with $F_{4}$ not given 
by Eq. (24). Background subtraction corresponds in our case to leaving out in Eq. (24) the terms that are factorizable. We are uncertain whether that correspondence is exact, since the experimental procedure for the determination of the background is not unique and involves steps that seem hard to duplicate in theoretical calculation. Our procedure of retaining only the non-factorizable component is the most sensible way to obtain the associated particle distribution, so we use

$$
F_{4}^{t r-b g}=(\mathcal{S T}+\mathcal{S S})_{13}(\mathcal{S T}+\mathcal{S S})_{24}
$$

which is not factorizable because of the correlation that exists between the shower parton at $q_{1}$ and the shower parton at $q_{2}$, as we have studied in Sec. 3 .

We note that Eq. (35) is a ratio of the integrals over the trigger momentum in contrast to the integral of the ratio that is calculated in [9]. Eq. (35) corresponds to the way that the data are presented in [5].

There is one piece of detail about the calculation using Eqs. (23) and (36) that we now discuss. Among the SPDs determined in [10] the gluon initiated quark distribution has a parametrization that leads to divergence, $G(x) \propto x^{-a}$, as the momentum fraction $x \rightarrow 0$. It is unphysical in that limit, although the parametrization renders a good fit of the fragmentation function away from that limit. In application to the calculation of $d N_{\pi} / p d p$, as in Eq. (10), the integral over $q_{1}$ is convergent, and at small $p$ the $\mathcal{S T}$ component is dominated by the $\mathcal{T} \mathcal{T}$ component. But when the background is subtracted with the $(\mathcal{T} \mathcal{T})(\mathcal{T} \mathcal{T})$ component removed from $d N_{\pi \pi} / p_{1} p_{2} d p_{1} d p_{2}$, the effect of large $G(x)$ at very small $x$ is exposed. Since that effect is unphysical and since we do not claim reliability of our formalism at very small 
$p_{T}$, we introduce an ad hoc factor

$$
s(q)=1-e^{-q / q_{0}}
$$

to suppress the distribution at small $q$ for all gluon initiated shower partons. With $q_{0}$ set at $1 \mathrm{GeV} / \mathrm{c}$ this procedure does not affect the results of our calculation for $p_{T}>1.5 \mathrm{GeV} / \mathrm{c}$, but does introduce a round-off at lower $p_{T}$ in the associated particle distribution. Figures in Sec. 4 all have that factor $s(q)$ included for gluon initiated showers.

Substituting Eq. (36) into Eq. (23) and then into Eq. (35), we obtain the results shown in Fig. 11 for (a) 0-10\% and (b) 80-92\% centralities. The calculated curves are for $\pi^{+}$trigger and $\pi^{+}$associated particle, while the data points from [5] are for all charged particles with 0-5\% centrality in (a) and for $p p$ collision in (b). The shapes of the $p_{T}$ dependence agree very well; the normalizations differ by a factor that can be attributed to the data being for all charged particles. Thus our formalism describes the correlation between particles in jets quite satisfactorily when compared to data.

Another area of possible comparison between theory and experiment is in correlation at low $p_{T}$. Data on a quantity denoted by $\Delta \rho / \sqrt{\rho}$ in Ref. [2] for $p p$ collision show a bulge plotted in transverse rapidity, defined by

$$
y_{t}=\ln \left[\left(m_{T}+p_{T}\right) / m_{\pi}\right]
$$

Since the quantity $\Delta \rho / \sqrt{\rho}$ is rather similar to our $G_{2}(1,2)$, it is of interest to display the latter in terms of $y_{t}$, which has the virtue of expanding the low $p_{T}$ scale. Since our calculations are unreliable for $p_{T}<1 \mathrm{GeV} / \mathrm{c}$, we consider only the region $y_{t}>2.5$. Instead of Eq. (13), we 
now define

$$
\rho_{1}(1)=\frac{d N_{\pi_{1}}}{d y_{1}}, \quad \rho_{2}(1,2)=\frac{d N_{\pi_{1} \pi_{2}}}{d y_{1} d y_{2}}
$$

where $d y_{i}=d p_{i} / m_{T} . \quad C_{2}(1,2)$ and $G_{2}(1,2)$ remain to be defined by Eqs. (12) and (16), respectively, but in terms of $y_{1}$ and $y_{2}$ they are changed in accordance to Eq. (39).

In Fig. 12 we show $G_{2}(1,2)$ as functions of $y_{1}$ and $y_{2}$ for (a) $80-92 \%$ and (b) $0-10 \%$ centralities. They are reproductions of the two surfaces in Fig. 10(a), now in terms of $y_{i}$. The upper ones in both are for peripheral collisions, while the lower ones are for central collisions. The increase at small $y_{1}$ and $y_{2}$ in Fig. 12(a) appears to agree with the bulge in the $p p$ collision data in [2] for $y_{1}, y_{2}>2.5$. For central collision Fig. $12(\mathrm{~b})$ has a peak and a dip, which are predictions that should be checked by the analysis of the corresponding data. For completeness, we show in Fig. 13 the $R_{C P}^{G_{2}}$ in the $y_{1}, y_{2}$ variables, in terms of which the dip becomes the dominant feature, even more prominent than in Fig. 10 (b).

\section{Conclusion}

We have calculated the single-particle distribution in $\mathrm{Au}+\mathrm{Au}$ collisions at all centralities, and considered various types of correlation functions in jets. For correlations between shower partons we have shown the drastic difference between the case when the hard parton momentum $k$ is fixed, as in $e^{+} e^{-}$annihilation, and the case in heavy-ion collisions where $k$ is not fixed. For hadron correlations we have considered three types of correlation fucntions, $C_{2}(1,2), K_{2}(1,2)$, and $G_{2}(1,2)$, and showed that $G_{2}(1,2)$ is best in displaying the structure of the dihadron correlation. 
We have found that the ratio of $G_{2}(1,2)$ for central collisions to that for peripheral collisions has a big dip in the $p_{1} \approx p_{2} \approx 2 \mathrm{GeV} / \mathrm{c}$ region. That is where the negative correlation between shower partons in a jet pulls down the hadronic $\rho_{2}(1,2)$ in central $\mathrm{Au}+\mathrm{Au}$ collisions relative to $\rho_{1}(1) \rho_{1}(2)$. However, in peripheral collisions $\rho_{1}(1) \rho_{1}(2)$ is so small compared to $\rho_{2}(1,2)$ that $C_{2}(1,2)$ remains positive for all $p_{1}$ and $p_{2}$. The calculated features of $G_{2}(1,2)$ can be checked by experiment, since all the quantities involved are directly measurable.

Although most of the results obtained on the correlation functions cannot be compared with any existing data, there is no basic hurdle to overcome for the data analysis to be carried out in ways that can check our prediction. The distribution of associated particles that we have calculated for $\pi^{+} \pi^{+}$correlation compares satisfactorily with the data except for the normalization difference due to the fact that the data are for all charged particles. Our result on $G_{2}$ for peripheral collisions has some resemblance to the data on $p p$ collisions, which are, however, not presented in a way that can facilitate quantitative comparison. Clearly, closer coordination between theoretical and experimental efforts would be of great value to both.

In this work the basic quantity is the correlation function $C_{2}(1,2)$ that is precisely defined. By subtracting $\rho_{1}(1) \rho_{1}(2)$ from $\rho_{2}(1,2)$, not only is the background subtracted, other factorizable terms are taken out too, creating the possibility that $C_{2}(1,2)$ can become negative. That possibility does not show up in the present experimental way of determining the associated particle distribution $[3,5]$, but may be present in other ways of studying correlations $[1,2,4]$. We suggest that future experimental work be directed toward determining the correlation function $G_{2}(1,2)$ and the central-to-peripheral ratio $R_{C P}^{G_{2}}(1,2)$. The absence of any hole in the experimental result on $R_{C P}^{G_{2}}(1,2)$, as shown in Figs. 10(b) and 
13, would provide valuable information on the nature of parton correlation that we have not incorporated in our formulation of the problem.

\section{Acknowledgment}

We are grateful to Tom Trainor and Fuqiang Wang for valuable communication on their work and to C. B. Yang for helpful comments. This work was supported, in part, by the U. S. Department of Energy under Grant No. DE-FG03-96ER40972.

\section{References}

[1] T. A. Trainor (STAR Collaboration), talk given at the 20th Winter Workshop on Nuclear Dynamics, Jamaica, 2004, hep-ph/0406116.

[2] R. J. Porter and T. A. Trainor, Acta Physica Polonica B 36, 353 (2005).

[3] D. Magestro, Proceedings of Hard Probes 04, Ericeira, Portugal (2004).

[4] J. Adams et. al., (STAR Collaboration), nucl-ex/0406035, 0408012, 0411003.

[5] J. Adams et. al., (STAR Collaboration), nucl-ex/0501016.

[6] R. C. Hwa and C. B. Yang, Phys. Rev. C 70, 024905 (04).

[7] R. C. Hwa and C. B. Yang, Phys. Rev. Lett. 93, 082302 (04); Phys. Rev. C 70, 037901 $(04)$.

[8] R. C. Hwa, C. B. Yang and R. J. Fries, Phys. Rev. C 71, 024902 (2005). 
[9] R. C. Hwa and C. B. Yang, Phys. Rev. C 70, 054902 (2004).

[10] R. C. Hwa and C. B. Yang, Phys. Rev. C 70, 024904 (2004).

[11] R. C. Hwa and C. B. Yang, hep-ph/0312271v1.

[12] B. A. Kniehl, G. Kramer and B. Pötter, Nucl. Phys. B597, 337 (2001).

[13] R. C. Hwa and C. B. Yang, Phys. Rev. C 66, 025205 (2002).

[14] D. K. Srivastava, C. Gale, and R. J. Fries, Phys. Rev. C 67, 034903 (2003).

[15] S. S. Adler et al (PHENIX Collaboration), Phys. Rev. Lett. 91, 072301 (2003).

[16] E. W. Weinsstein, Correlation Coefficient, MathWorld - A Wolfram Web Resource. http://mathworld.wolfram.com/CorrelationCoefficient.html.

[17] R. J. Fries, S. A. Bass, and B. Müller, Phys. Rev. Lett. 94, 122301 (2005).

[18] R. C. Hwa and C. B. Yang, Phys. Rev. C 67, 034902 (2003).

[19] R. J. Fries, B. Müller, C. Nonaka, and S. A. Bass, Phys. Rev. Lett. 90, 202303 (2003); Phys. Rev. C 68, 044902 (2003).

[20] A. Majumder, E. Wang and X. N. Wang, nucl-th/0412061. 


\section{Figure Captions}

Fig. 1. Single-particle distributions of produced $\pi$ in $\mathrm{Au}+\mathrm{Au}$ collisions at $\sqrt{s}=200 \mathrm{GeV}$ for four centrality cuts. Data are from [15], and the solid lines are the results of calculation in the recombination model.

Fig. 2. The three parameters $C, T$ and $\xi$, determined by fitting the data at all centralities.

Fig. 3. The single-particle distributions for (a) most central and (b) most peripheral collisions, showing the three components that contribute to the sums in solid lines.

Fig. 4. (Color online) The ratio $r_{2}(1,2)$ in terms of the shower parton momentum fractions $x_{1}$ and $x_{2}$ for a hard gluon momentum fixed at a large values $k$.

Fig. 5. (Color online) The ratio $r_{2}(1,2)$ in terms of the shower parton momenta $q_{1}$ and $q_{2}$ in heavy-ion collisions for two extreme centralities.

Fig. 6. The correlation functions $C_{2}$ for $\pi^{+} \pi^{+}$in HIC, showing the contributions from the three combinations of $\mathcal{S}$ and $\mathcal{T}$ partons for (a) central and (b) peripheral collisions along the diagonal where $p_{1}=p_{2}$.

Fig. 7. (Color online) The correlation functions $C_{2}$ for two centralities when they are positive.

Fig. 8. The normalized correlation functions $K_{2}$ along the diagonal where $p_{1}=p_{2}$ for (a) central and (b) peripheral collisions. The partially normalized correlation functions $G_{2}$ for (c) central and (d) peripheral collisions. 
Fig. 9. A comparison of the three types of correlation functions $C_{2}, K_{2}$ and $G_{2}$ in semi-log plots, with the gap corresponding to where the functions are negative.

Fig. 10. (Color online) (a) $G_{2}(1,2)$ plotted as functions of $p_{1}$ and $p_{2}$ for two centralities; (b) the ratio $R_{C P}^{G_{2}}$ of central to peripheral $G_{2}$.

Fig. 11. Associated particle distributions of $\pi^{+} \pi^{+}$for (a) central (0-10\%) collisions compared to the STAR data [5] for all charged particles at (0-5\%) centrality and (b) peripheral (80-92\%) collisions compared to the STAR data [5] for all charged particles in $p p$ collisions.

Fig. 12. (Color online) $G_{2}(1,2)$ for $\pi^{+} \pi^{+}$plotted in terms of transverse rapidities for (a) peripheral (80-92\%) and (b) central (0-10\%) collisions.

Fig. 13. (Color online) The ratio $R_{C P}^{G_{2}}$ in transverse rapidities. 


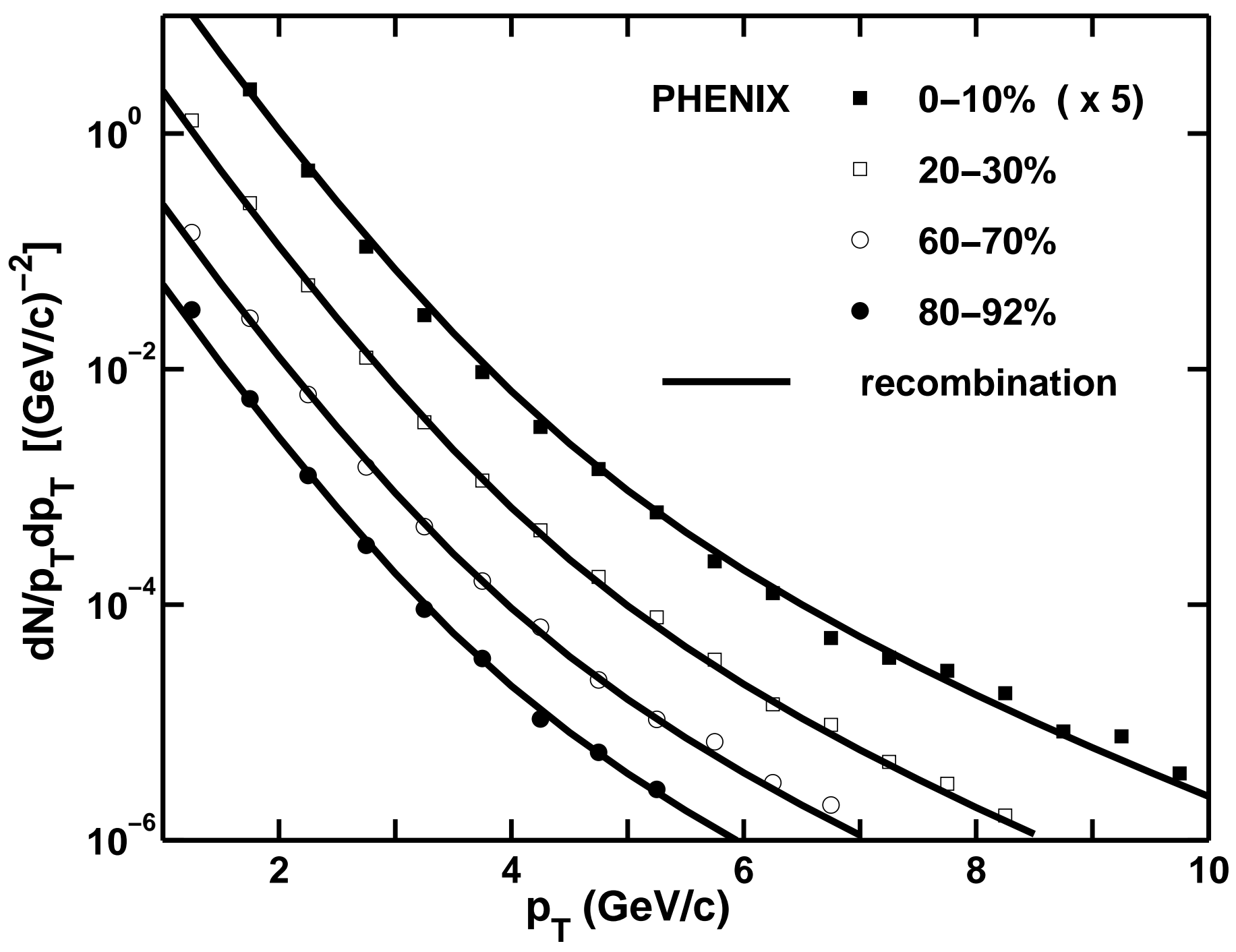




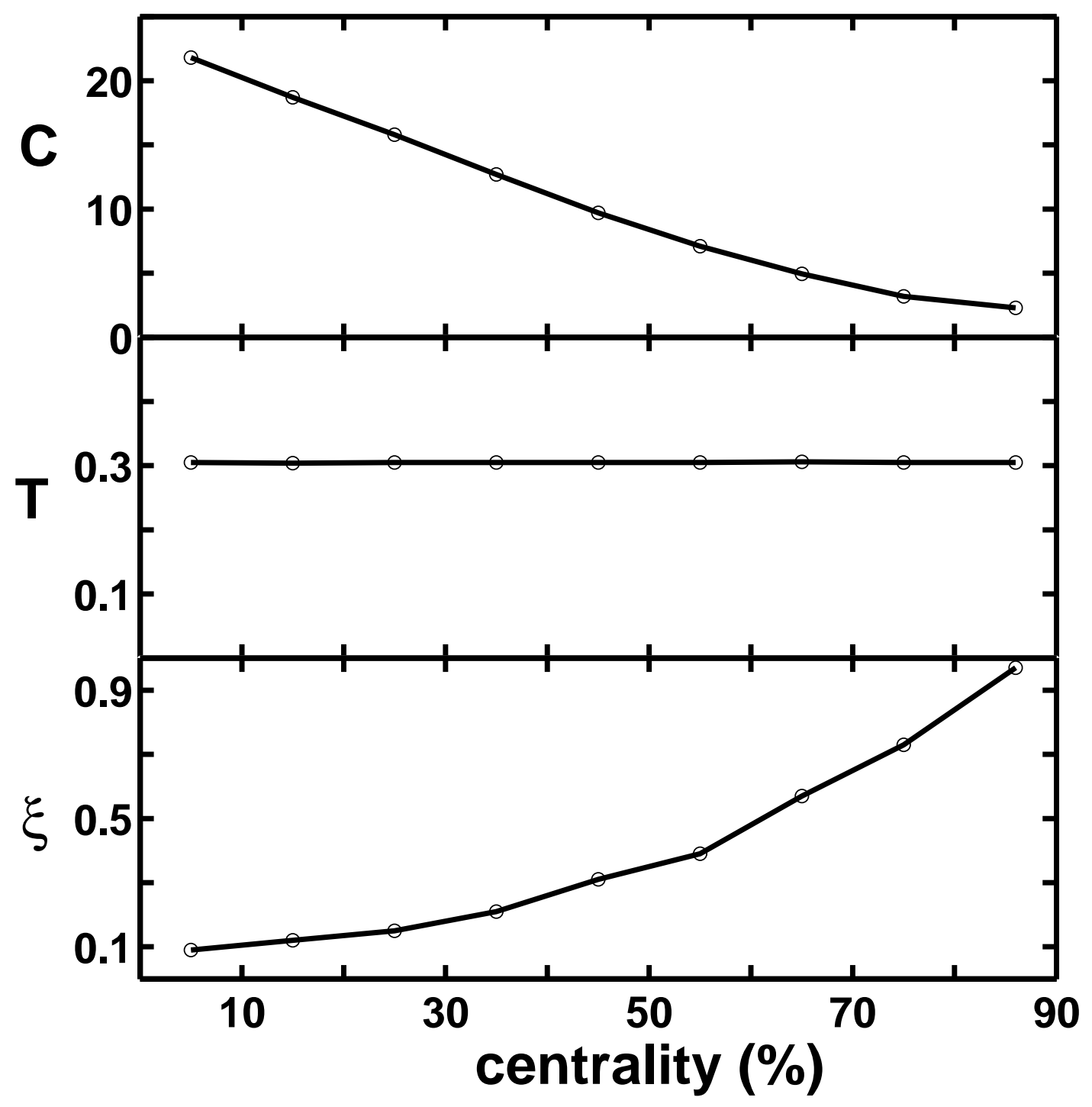




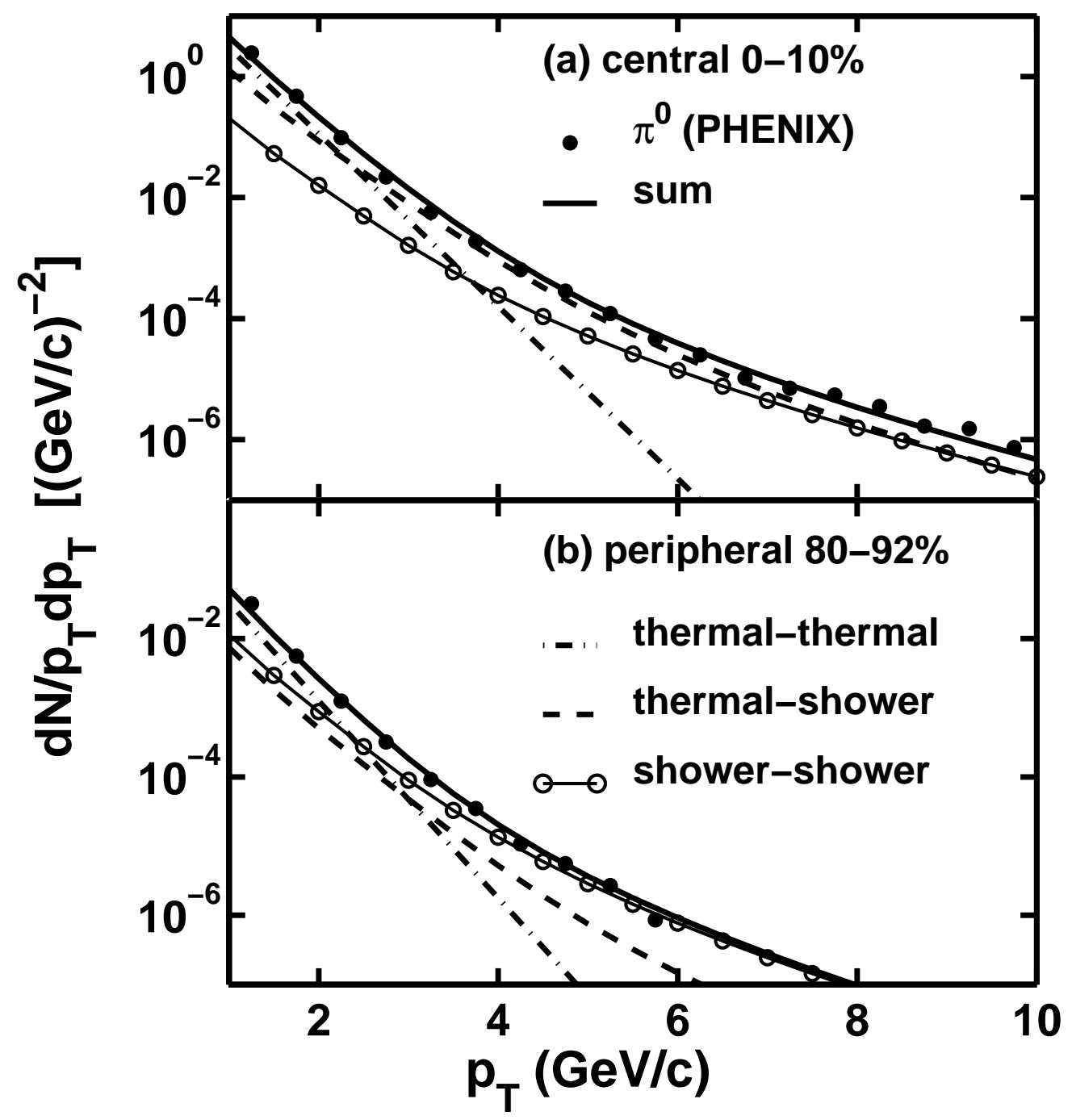




\section{Shower partons with fixed $k$}

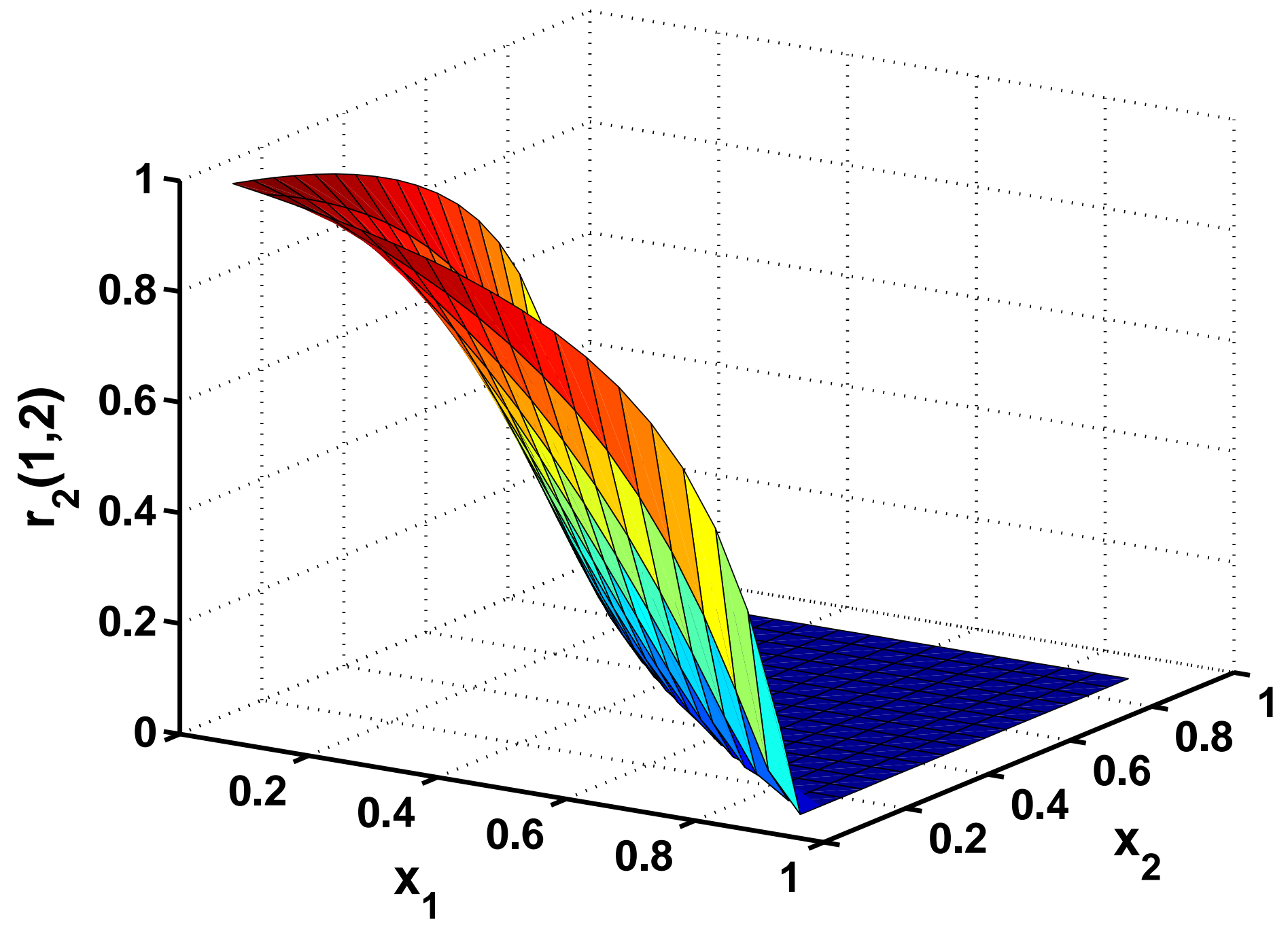




\section{Shower partons in HIC}

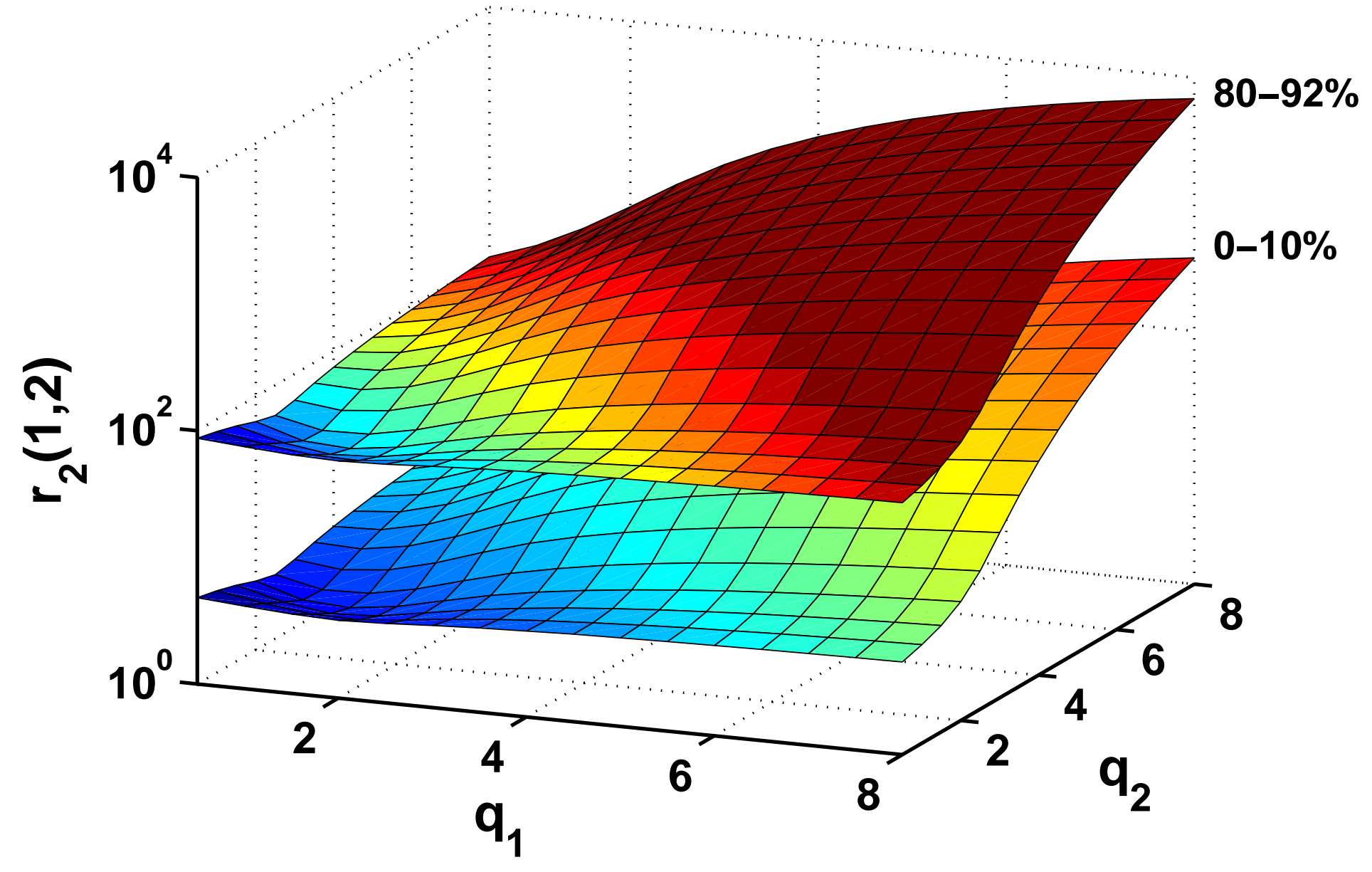



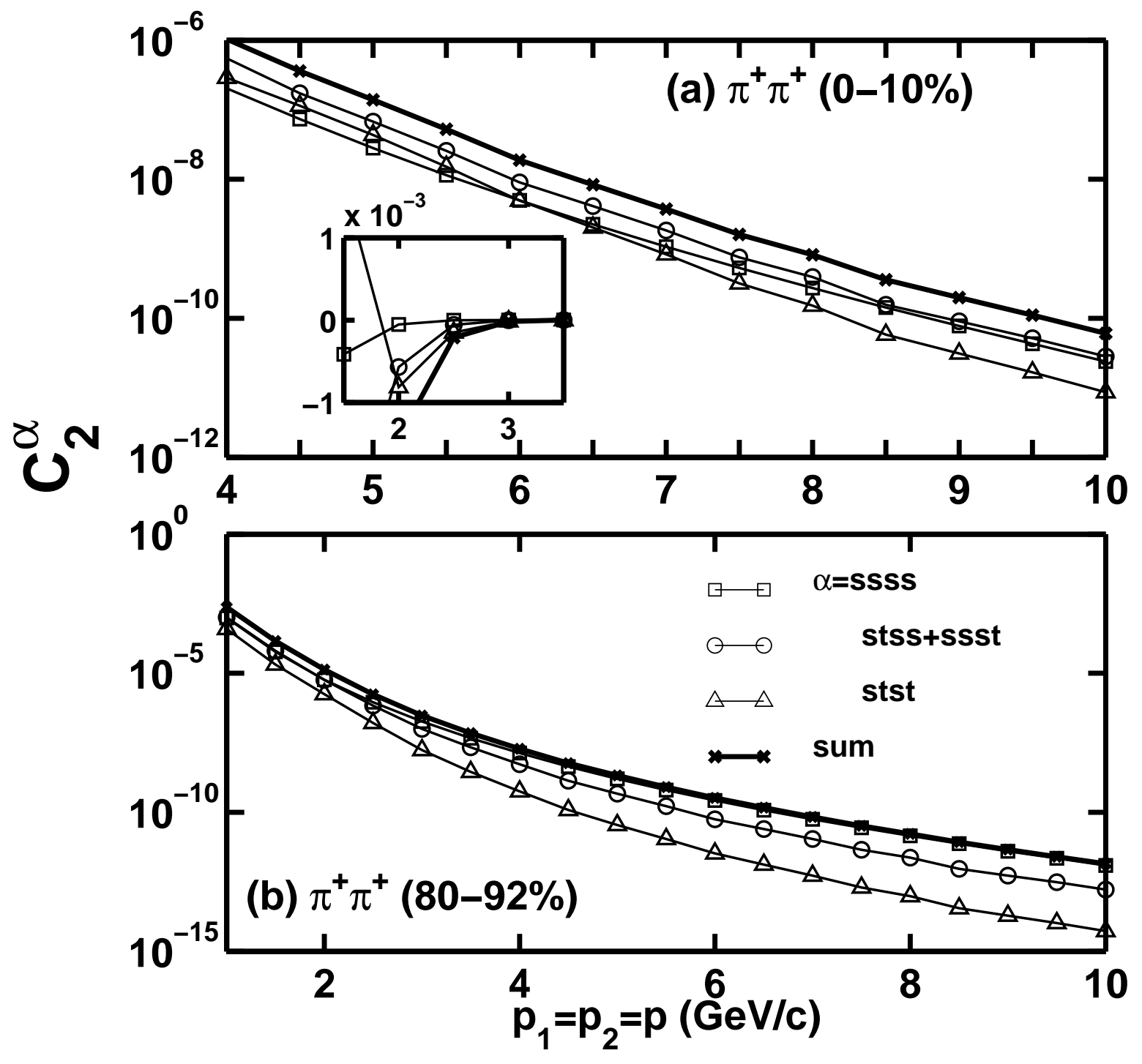


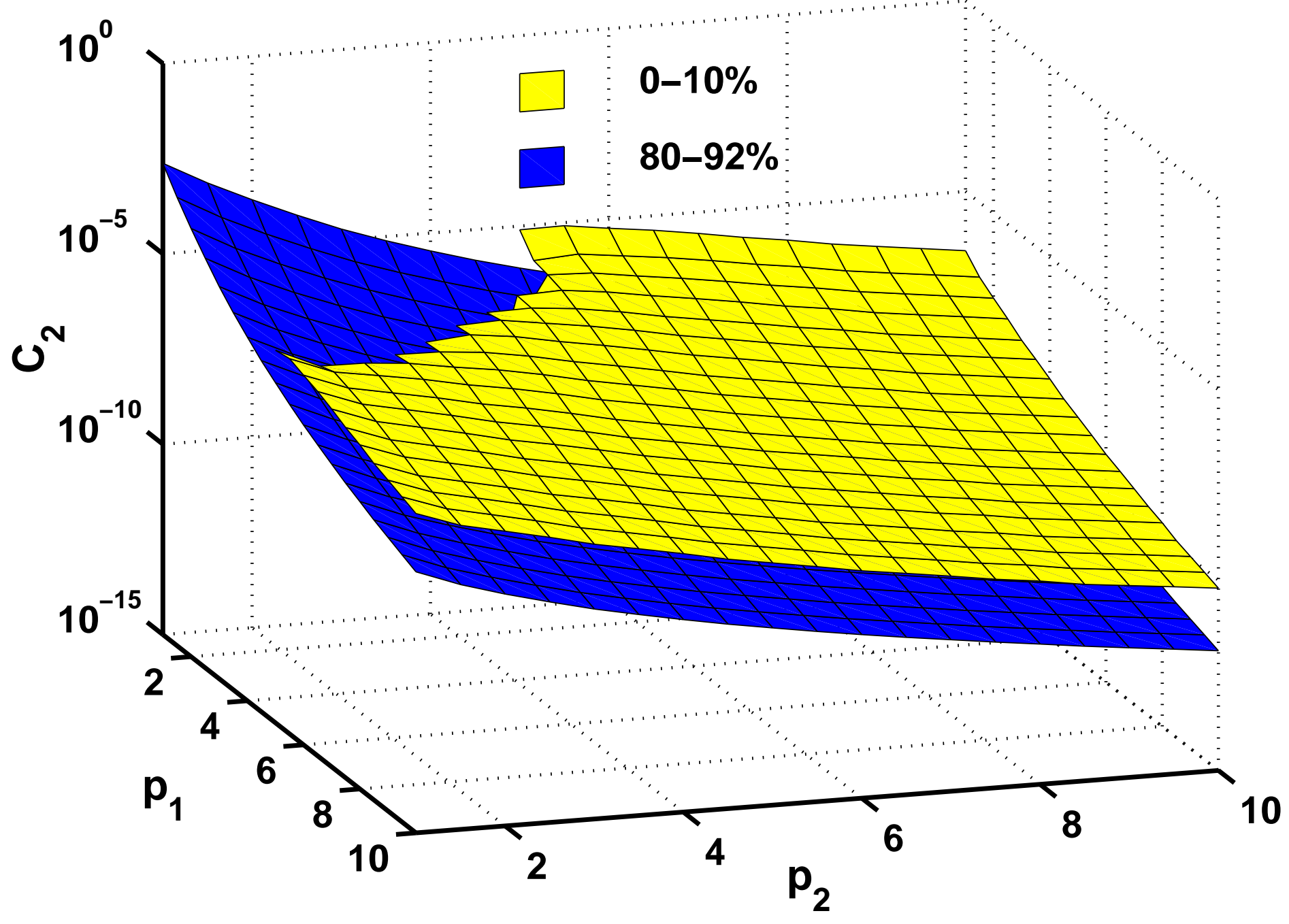



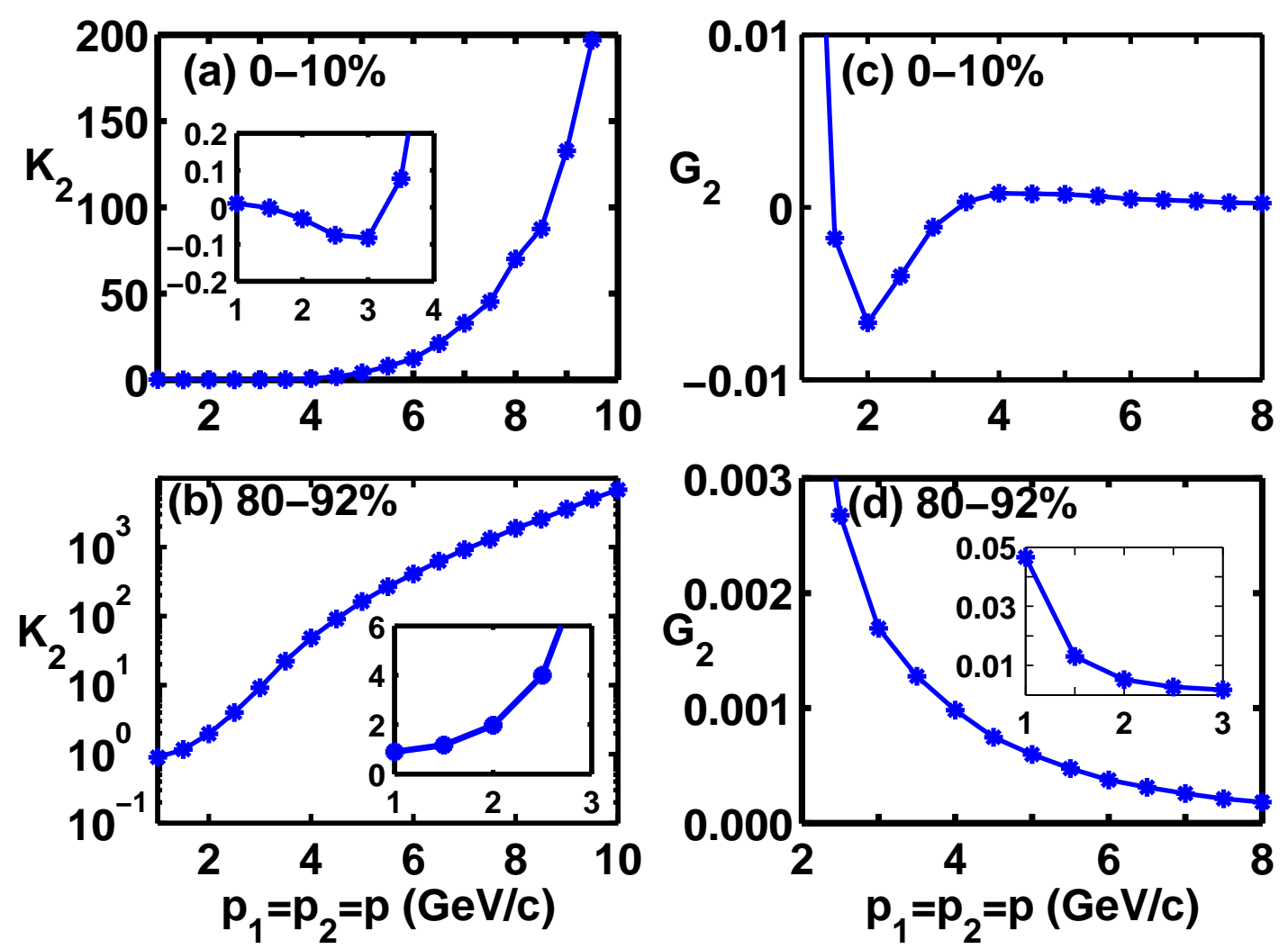


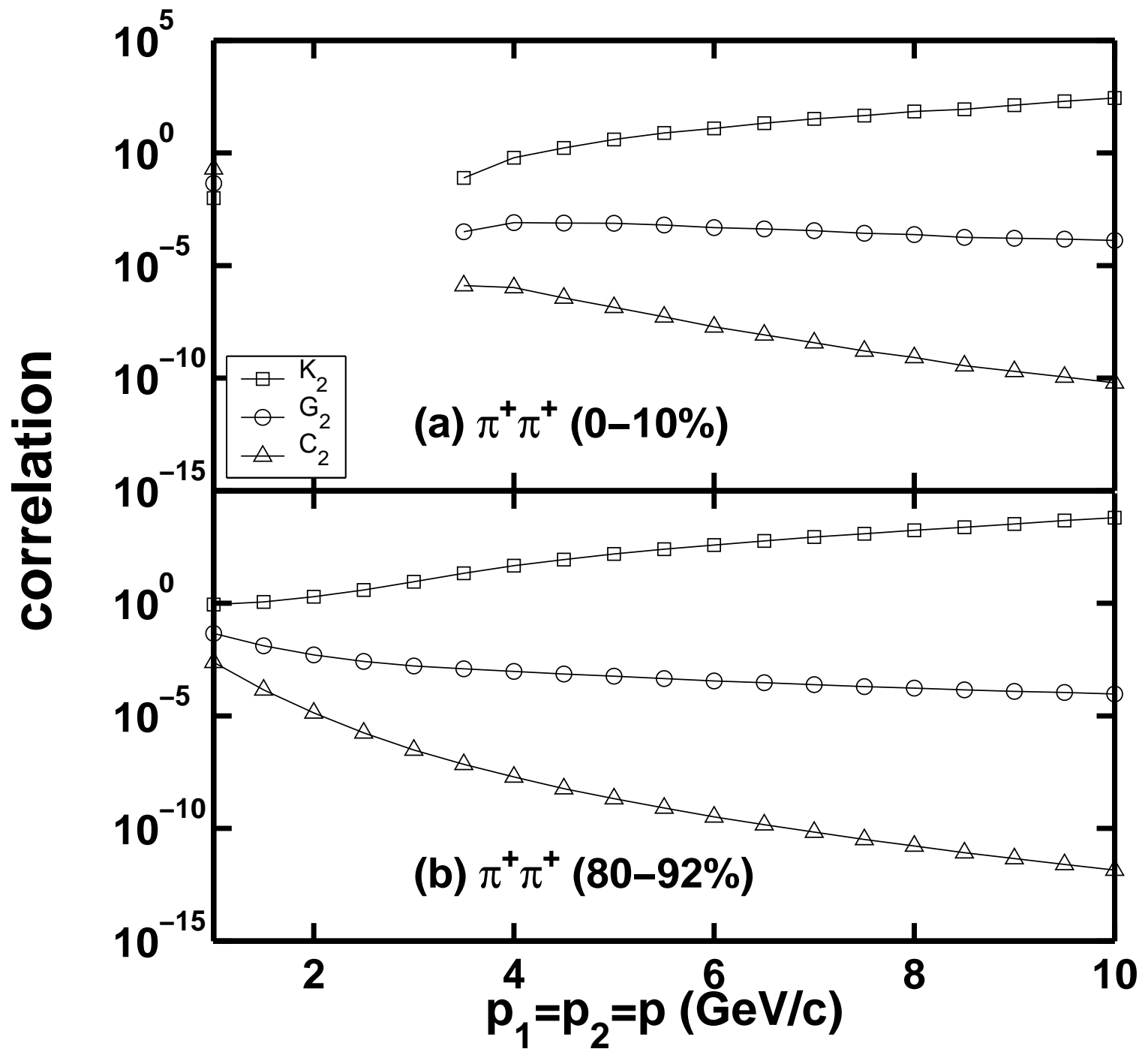



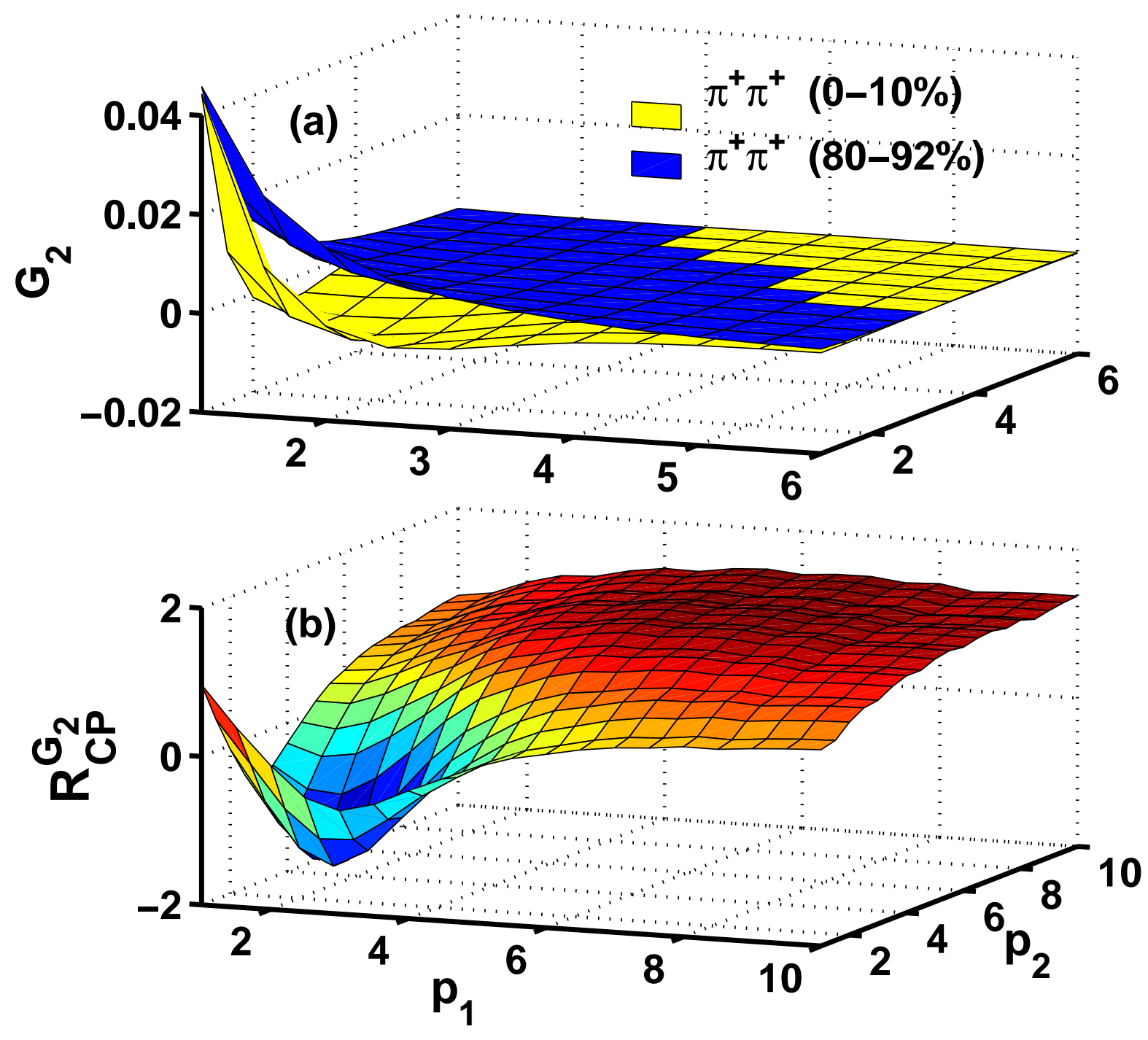


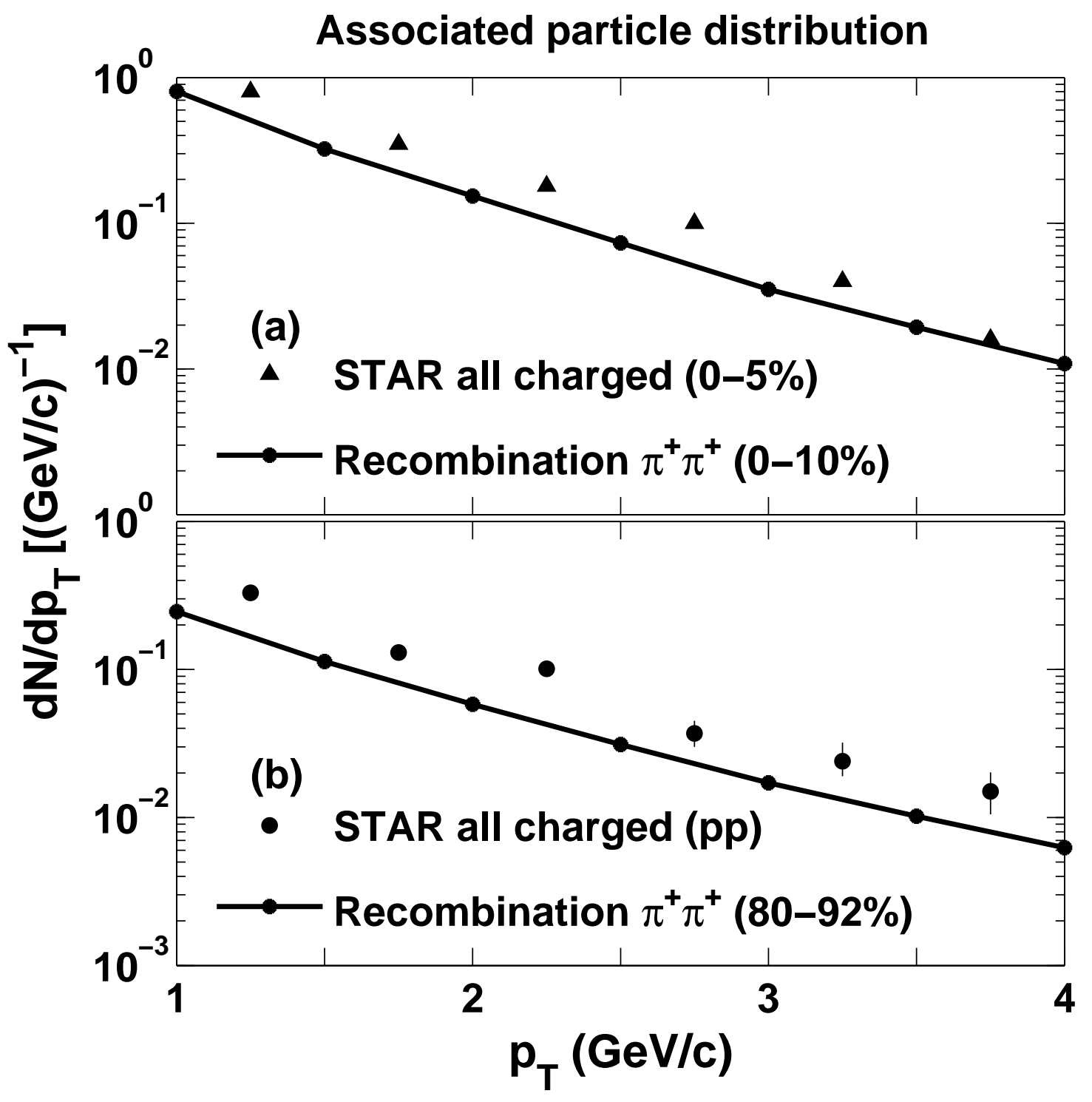



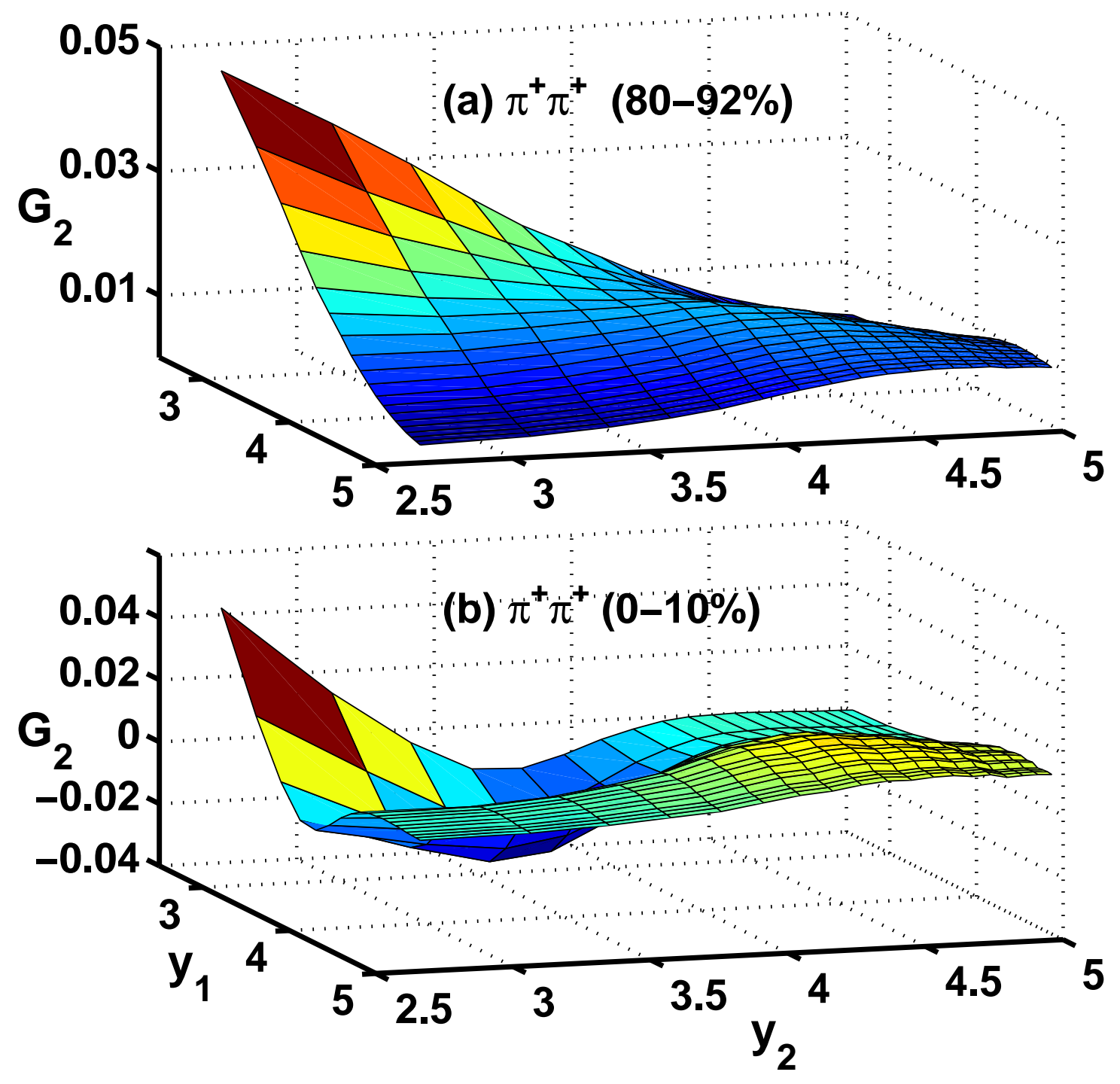


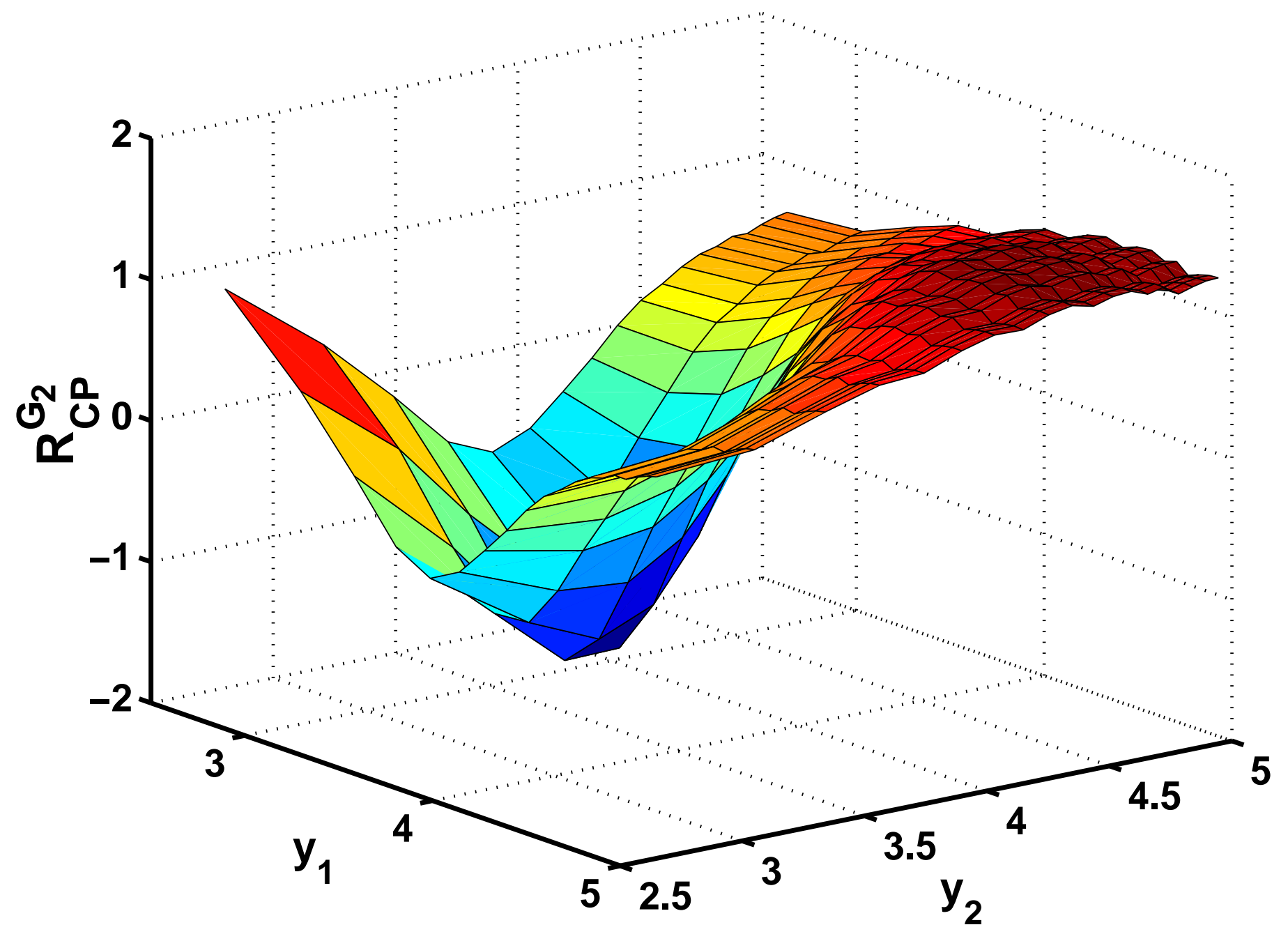

